\title{
Cultura Cultura
}

Revistade historia e Teria das ldeais $\quad$ Revista de História e Teoria das Ideias

vol. 38 | 2019

Bibliotecas públicas, políticas culturais e leitura pública

\section{Alfabetización crítica y lectura digital}

\section{Margarita Pérez Pulido}

\section{(2) OpenEdition}

Journals

Edición electrónica

URL: https://journals.openedition.org/cultura/5499

DOI: $10.4000 /$ cultura. 5499

ISSN: 2183-2021

Editor

CHAM - Centro de Humanidades

\section{Edición impresa}

Fecha de publicación: 31 diciembre 2019

Paginación: es 105-121

ISSN: 0870-4546

\section{Referencia electrónica}

Margarita Pérez Pulido, «Alfabetización crítica y lectura digital», Cultura [Online], vol. 38 | 2019, posto online no dia 02 janeiro 2021, consultado o 25 julho 2021. URL: http://journals.openedition.org/ cultura/5499 ; DOl: https://doi.org/10.4000/cultura.5499

Este documento fue generado automáticamente el 25 julio 2021.

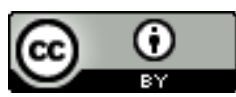

Cultura is licensed under a Creative Commons Atribuição 4.0 International. 


\title{
Alfabetización crítica y lectura digital
}

\author{
Margarita Pérez Pulido
}

\section{Introducción}

El objetivo de este trabajo es analizar los conceptos de alfabetización crítica y lectura crítica en el contexto del término Lifelong Learning o Aprendizaje a lo largo de la vida, uno de los puntos principales de los Objetivos de Desarrollo Sostenible (ODS) que contiene la Agenda 2030 de la ONU. El acceso a la información se ha convertido en uno de los 17 nuevos ODS a nivel global. En el documento de IFLA denominado "Las bibliotecas y la implementación de la Agenda 2030 de la ONU” (IFLA 2015) se parte del concepto de alfabetización universal para asegurar el acceso público a la información como parte en concreto del ODS 4.

3 Este término de Lifelong Learning o Aprendizaje a lo Largo de la Vida, aparece ya mencionado en la Declaración de Hamburgo de 1997, por la que UNESCO e IFLA se unen en la creencia de que las bibliotecas, además de museos y otras organizaciones, deben adoptar un papel esencial para el aprendizaje a fin de crear ciudadanos modernos. Con respecto a las bibliotecas, en el año 2000 surge un proyecto que culmina en 2003 con la creación de una sección de IFLA para la Alfabetización (IFLA 2004). La definición de alfabetización de IFLA-UNESCO que aparece en este documento, se refiere al aprendizaje en diferentes entornos, en diversos temas y centrado en la enseñanza de la búsqueda de información y otras cuestiones relacionadas con la inclusión social. El Memorándum de Lisboa (2000) ya especifica para el ámbito europeo cuáles son los aprendizajes que se unen a la búsqueda de información como parte del término denominado alfabetización: el aprendizaje de lenguas, de nuevas tecnologías, habilidades sociales y emprendimiento. Para ello, se deben organizar redes de cooperación con diversas instituciones, en las que las bibliotecas ocupan un papel determinante en la utilización de espacios, gestión de la colección, protección de la herencia cultural, y búsqueda de información para la participación democrática. En 2010 se crea un sitio llamado "InfolitGlobal", con la participación de IFLA/UNESCO, en donde se establecen por primera vez políticas internacionales y se crean contenidos de 
aprendizaje (Pasadas 2010).Como consecuencia de esta necesidad de impulsar el aprendizaje a lo largo de la vida, se irán creando alianzas entre instituciones, en las que la biblioteca continuará teniendo un papel importante yse verá inmersa en políticas, normas y acciones tanto a nivel local, como nacional e internacional.

De esta forma, por medio de un trabajo teórico basado en la recopilación de fuentes bibliográficas y descripción de ejemplos más significativos, nos proponemos analizar, en primer lugar, el contexto de la alfabetización en la actualidad, en una evolución del concepto y su significado a lo largo de los últimos años, en que la diversidad de términos utilizados para mencionar la misma actividad ha variado con el tiempo en función de la evolución del uso de las nuevas tecnologías en la educación y en la información en general. De esta manera, llegamos hasta el concepto de alfabetización crítica, y en consecuencia, lectura crítica, como un fenómeno imprescindible hoy día en nuestra sociedad, y como una característica global e indispensable del entorno digital.

\section{Contexto de la alfabetización en la actualidad}

6 Para definir el contexto de la Alfabetización en la actualidad debemos hacer referencia al término de Lifelong Learning o Aprendizaje a lo largo de la vida, como ya hemos mencionado anteriormente. La UNESCO es una organización activa en este sentido. En 2016 crea el "Instituto UNESCO para el Lifelong Learning" (UNESCO 2016), en el que las bibliotecas están muy presentes, ya que contempla la colaboración de las mismas en iniciativas nacionales y regionales, que parten de otras instituciones que poseen las ciudades relacionadas con la educación fundamentalmente. En este sentido, se plantea como necesaria la colaboración de las bibliotecas en iniciativas nacionales y regionales, en la atención especial a nuevos públicos como los niños, la familia en la lectura en común, los jóvenes y adultos en el trabajo y otras necesidades cotidianas, haciendo especial énfasis en la inclusión de grupos vulnerables. Otra circunstancia nueva es la insistencia en la recogida de datos para la planificación y la evaluación.

7 El Movimiento Learning Cities cuyo origen se encuentra igualmente en la UNESCO a partir de 2015 (UNESCO 2015), tiene como objetivo fundamental la creación de ciudades sostenibles en el aprendizaje de los ciudadanos (Smart Cities) a partir de la creación de una estructura coordinada de todas las partes interesadas en una ciudad concreta, estableciendo planes de acción a nivel local. A su vez, se crea la Red Mundial de las Learning Cities (GNLC) en donde se visibilizan proyectos llevados a cabo para una futura cooperación conjunta entre ciudades.

8 De la consideración del concepto de Lifelong Learning o Aprendizaje a lo largo de la Vida podemos mencionar las estrategias que se están llevando a cabo en nuestro país (España). El Ministerio de Educación, Cultura y Deporte, a través de la Subdirección General de Aprendizaje a lo Largo de la Vida, ha creado el "Plan Estratégico de Aprendizaje a lo Largo de la Vida" (2014) que incluye los objetivos que hemos mencionado de la UNESCO. Así, establece prioridad en grupos de interés como los jóvenes y ancianos, resalta la importancia de la recolección de datos y las técnicas de evaluación con indicadores para la medida de resultados, el desarrollo de competencias para conseguir una ciudadanía activa, y el desarrollo personal de todos los que forman parte de la sociedad en general. En el caso español, debemos resaltar, además, la publicación de la Ley Orgánica 3/2018, 5 diciembre, de Protección de Datos Personales y Garantía de los Derechos Digitales (2018), en cuyo Título X, se hace referencia, por primera vez, a la alfabetización como un derecho digital. 
9 Por otra parte, las Comunidades Autónomas también han establecido sus planes de acción sobre alfabetización como parte importante del concepto de aprendizaje a lo largo de la vida. La Comunidad Autónoma de Andalucía creó el "Plan ALBA" (2011) para establecer una política de alfabetización digital en la Región, implantando la Certificación de competencias informáticas y de la información, y el Programa "Andalucía Compromiso Digital" (ACD) para los refugiados. Otro ejemplo es la Comunidad Autónoma del País Vasco, que publicó la Ley 1/2013 de aprendizaje permanente (2013), en la que se menciona el aprendizaje a lo largo de la vida como un derecho de la ciudadanía vasca, en su triple tipología de aprendizaje (formal, no formal e informal). Esta ley hace énfasis en cuestiones como el acceso al trabajo, la integración social y la preparación para el envejecimiento activo de las personas adultas. Es interesante también destacar de esta ley la consideración de los llamados "Servicios de información y orientación", entre los que menciona a las bibliotecas como instituciones de cooperación con otras para conseguir estos objetivos.

Otros instrumentos muy interesantes para el desarrollo del concepto de Alfabetización son los estudios de prospectiva que elaboran los diferentes organismos a nivel nacional y global como estrategia fundamental para el futuro. De este modo, en España podemos hablar del informe titulado "Prospectiva 2020" (GRUPO 2013) cuyo punto 8 relaciona la educación, aprendizaje y habilidad con la misión de la biblioteca y su aplicación práctica, sugiriendo acciones para llevar a cabo procesos de diversos tipos de alfabetización, a la vez que considera el aprendizaje como un servicio más de la biblioteca. El Aprendizaje a lo largo de la vida como estrategia fundamental para el futuro también lo encontramos en el informe de prospectiva de la IFLA (2013) en el que su apartado "ambiente tecnológico" menciona, como elementos clave de futuro, la educación global y móvil. Este punto consiste en la disponibilidad de recursos educativos en línea y los Massive Open Online Courses (MOOCs), la preservación de contenidos locales, patrimonio cultural y digital, el acceso igualitario a la información y lucha contra la censura en internet, el respeto a los derechos de propiedad intelectual, la figura del bibliotecario como intermediario (soporte, co-estudio, tutor), y el crecimiento de la participación de los ciudadanos en el universo digital.

11 Otros trabajos de este tipo ayudan a definir el concepto de aprendizaje a lo largo de la vida en general y de alfabetización en concreto. Es el caso del estudio de la Federación Española de Sociedades de Archivística, Biblioteconomía, Documentación y Museística (FESABID), elaborado por su Grupo de Trabajo de Identidad y Posicionamiento Profesional, sobre el perfil del consumidor de información en España (FESABID2014) respecto a las tendencias en consumo de información global y la relación de los profesionales con el sector de la información. En este estudio aparecen los puntos principales que caracterizan en general a este consumidor español de información independientemente del segmento de edad o laboral al que pertenezca:

Son usuarios intensivos en redes sociales;

Hacen blogs sobre todo para su proyección personal;

Se mantienen conectados a datos y publicaciones online para su trabajo;

Usan las redes sociales para conexiones con amigos;

Son adictos a las redes sociales para mantenerse informados;

Su uso es inmediato, visual, simple, y gratuito;

Conceden gran relevancia a las opiniones de los demás;

Se entremezclan la vida personal y profesional en la conexión a internet; Se superan las barreras de inseguridad. 
Estas características resultan muy valiosas para conocer qué tipo de alfabetización se debe priorizar y, en general, qué acciones previsibles deben llevarse a cabo por parte de las diferentes instituciones, como ya hemos mencionado anteriormente y, en definitiva, para encontrar una definición del alfabetización más amplia y actual acorde con las características sociales e informacionales de los ciudadanos para una sociedad democrática. Multialfabetización (Multiliteracy), como el conjunto de todas las alfabetizaciones en cualquier situación de la vida diaria y a cualquier edad de una persona. Según este autor, la definición incorpora, para cualquier acción, cualquier tipo de soporte y lenguaje, y la persona aprende según su personalidad y contexto, aplicando un modelo en el que se encuentran presentes cinco enfoques: representacional, educacional, social, organizacional, intertextual e ideológico. Esta multialfabetización comprende la alfabetización informacional, digital, básica, mediática, jurídica, tecnológica, multicultural, emocional sobre cualquier asunto o materia, en un aprendizaje autónomo de acuerdo a un nivel y exigencia, donde la biblioteca actúa como vínculo entre el aprendizaje y la persona, y cuya finalidad es fundamentalmente crear ciudadanos libres y críticos. Por su parte, Sánchez-García y Yubero (2015) establecen una nueva tipología de alfabetizaciones: informacional, en la búsqueda de información, digital en el uso de nuevos recursos y tecnologías, y mediática en las nuevas formas de participar y comunicar en la red. Sturges y Gastiner (2012) establecen otra tipología de alfabetizaciones interesante en su evolución, acorde con las necesidades de la nueva sociedad: mediática, o la interpretación de la información recibida por los medios de comunicación; informática, o la formación en usos de programas informáticos y aplicaciones del ordenador; digital, para el uso de la tecnología digital y los móviles; alfabetización web o la interpretación del contenido en la web. A esta tipología añaden, la alfabetización cívica, o la interpretación de mensajes y propaganda ideológica y política; y la alfabetización crítica, o la adquisición de capacidad crítica para entender el contenido, contexto e ideología de una determinada noticia, texto o idea. Para esto, proponen un nuevo término "alfabetización informacional crítica".

Haciendo una evolución en el tiempo podemos considerar varios conceptos y términos relacionados con el aprendizaje a lo largo de la vida y a la alfabetización en general. De Llegados a este punto, queremos detenernos en los conceptos de alfabetización mediática e informacional, y en el de New Media Literacy, por considerar que están relacionados con más intensidad con lo que vamos denominar alfabetización crítica.

Gutiérrez y Tyner (2012) se refieren a la alfabetización mediática o digital como aquella que se dedica a la educación en los medios, lo audiovisual, lo digital, lo multimodal. Para estos autores, la alfabetización mediática supone la capacidad de obtener una comprensión y crítica de la naturaleza de los medios de comunicación de masas, y el objetivo es capacitar para un uso crítico de las nuevas tecnologías. Reconocen la existencia de una confusión terminológica, como ya hemos visto en otros autores, e identifican dos campos convergentes o tendencias que son las alfabetizaciones mediática e informacional. Aún así, establecen alguna diferencia entre ambas, por la que la alfabetización digital está más relacionada con la tecnología y la alfabetización mediática con la presencia de los medios en nuestra sociedad. Consideran la alfabetización crítica una característica fundamental de ambas alfabetizaciones. En 
opinión de estos autores, la alfabetización del siglo XXI se caracteriza por ser mediática, digital, multimodal, crítica y funcional.

Respecto al concepto de este tipo de alfabetizaciones mediática e informacional, encontramos dos posturas interesantes. La primera de ellas, se refiere a la "postura integradora" de la UNESCO (Gutiérrez y Tyner 2012), que comprende nuevas dimensiones de la alfabetización: la mediática, o la importancia de los medios de comunicación; la digital, o el manejo de la información digitalizada; y la multimodal, o la habilidad para combinar imagen, texto, sonido. La característica resultante de esta unión nos lleva a lo que se ha denominado "pensamiento crítico". Por otra parte, la propuesta de la OCDE (García Moreno y Hernández-Pérez 2010) habla de tres dimensiones: la dimensión de la información, la fuente y el producto; la dimensión de la comunicación, colaboración e interacción virtual; y finalmente, la dimensión ética e impacto social, o la implicación social, económica, cultural, que nos conduce igualmente al pensamiento crítico. En Europa, la llamada "Carta Europea para la Alfabetización Mediática" (2005) surge, por parte de las organizaciones adheridas, para apoyar el establecimiento de la información mediática en todo el continente, respaldar una definición específica, comprometerse a tomar medidas para su desarrollo y crear redes europeas de trabajo.

Finalmente, debemos tomar en consideración el concepto de New Media Literacy que aparece bien definido por Borges y Marzal (2017). Estos autores se refieren a este término como la unión de alfabetización informacional, el conocimiento y uso de fuentes de información por una persona pero sin ser productora; la alfabetización digital o mediática, por la cual una persona crea, distribuye contenidos y los comunica; y el consumo y producción de información generada en medios sociales.

En consecuencia, según estos autores, se necesitan nuevas competencias de aprendizaje:

Capacidad crítica sobre el contenido de los medios de comunicación de masas;

Interacción entre las personas;

Comunidades virtuales de aprendizaje;

Lectura colaborativa;

Capacidad de participar interactivamente y críticamente en ambientes mediáticos;

Enlazar los valores éticos y sociales con la construcción crítica de contenidos.

Así pues, el objetivo de la alfabetización informacional, continuando con la explicación de Borges y Marzal (2017), es la competencia crítica, es decir, crear un consumidor crítico en la comprensión de tipos y formatos de información, el ejercicio del análisis crítico en estos diferentes tipos y formatos de información, y la evaluación de información digital, sin ser necesaria la creación de contenidos. Sin embargo, la alfabetización digital crea un ciudadano crítico en los contenidos que consume y en su comunicación, con competencias en comunicación, creación de contenidos, y relaciones con otras personas. $Y$ en este contexto surge una serie de competencias que los autores denominan "nueva lectura", es decir, una lectura colaborativa, que crea conocimiento asociativo relacionado con espacios digitales, y un aprendizaje descentralizado en la creación de las llamadas comunidades digitales de aprendizaje como espacios abiertos para el aprendizaje. Este nuevo concepto de alfabetización le da a la persona la capacidad de participar en ambientes mediáticos de manera crítica y en la creación de contenidos, donde los valores sociales y éticos están siempre presentes.

3. Alfabetización crítica y lectura crítica 

alfabetización denominada "Alfabetización crítica". Según Serrano de Moreno y Madrid de Forero (2007), este término se considera una característica de la alfabetización mediática y digital que consiste básicamente en la capacidad de desempeñar funciones sociales como miembro de una comunidad en el contexto de una determinada cultura. Igualmente, ayuda a comprender la realidad, cuestionarlos valores dominantes, descubrir intenciones e ideologías en diversos productos culturales, y conseguir la capacidad del compromiso crítico con textos y tecnologías para la interpretación social de la realidad.

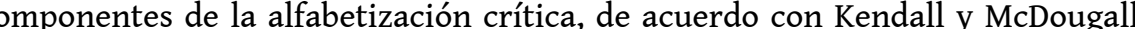
(2012), nos dirigen hacia la capacidad de descifrar el código de los textos, participar en la comprensión de significados; usar textos en variados contextos culturales y sociales; y a analizar críticamente los textos, con una cualidad extremadamente importante, en su opinión, que es el énfasis en la idea de que los textos no son ideología neutral sino que representan las ideas del autor. De acuerdo a estos autores, por tanto, un proyecto de alfabetización crítica abarcaría, además del aspecto tecnológico, el texto o textos elegidos y su análisis con unos parámetros de seguimiento: la forma en que el texto y persona conectan, la influencia en la identidad del individuo fuera y dentro de la red, el análisis del lenguaje, y la atribución de significado al texto y a la persona misma.

En la línea que acabamos de explicar, destacan proyectos europeos sobre educación crítica, como el llamado "Educaunet" sobre educación para los riesgos asociados a internet para jóvenes de 8 a 18 años, llevado a cabo durante la década del 2000, en el que participó Portugal junto a otros 6 países europeos, en concreto, desde la Universidad del Algarve (Reia-Baptista 2006). Este proyecto consistió en elaborar un kit de materiales pedagógicos para investigar sobre los hábitos de los jóvenes en este nuevo contexto. Uno de estos países participantes, Bélgica, ha seguido desarrollando interesantes proyectos, como el llevado a cabo por la Universidad de Lovaina (Fastrez, Thibault, Bilhl y T'Kint 2019), para países de lengua francófona, sobre la capacidad de los jóvenes para acceder a los medios, comprender y evaluar críticamente los contenidos y crear mensajes en varios contextos. Igualmente, de otro de los países participantes en este tipo de proyectos europeos, Francia, podemos obtener ejemplos interesantes. En el sitio web del Ministère de l'Éducation Nationale et de la Jeunesse, llamado "Éduscol" (2019), puede encontrarse una selección de sitios especializados de cuestiones de interés educativo en diferentes medios, sobre aspectos legales y actitud responsable y cívica, o sobre herramientas y uso de internet. Además, se encuentra todo tipo de informes, por ejemplo, sobre los beneficios de la lectura digital para familias vulnerables en lugares de crisis, realizado en 2019, o el informe de la UNESCO sobre lectura en la era mobile de 2010, entre otros. En este país se habla de un neologismo "Illectronisme" (Desjardins, 2000) para referirse a la falta de conocimiento sobre el uso de los recursos electrónicos, su práctica y el contenido y verificación de la información. Relacionado con este segundo punto, destaca igualmente el informe sobre las Fake News y la alfabetización crítica realizado por la Commission on Fake News and the Teaching of Critical Literacy in Schools, en el Reino Unido (Fake 2018). Partiendo de una definición de alfabetización digital, se explica el papel de la alfabetización crítica y cómo deben desarrollarse herramientas para que los niños sean capaces de identificar las fake news y su impacto en la sociedad. 

concepto de alfabetización crítica. ONDULA (2019) tiene como objetivo principal promover el pensamiento crítico, creativo y ético de la cultura digital en familias y en la infancia y juventud. Para ello, desarrolla diversos materiales y publicaciones, entre las que destacan una "Guía de alfabetización digital crítica", que anima a acercarse a la cultura digital de una forma muy comprensible y pedagógicamente muy buena, y un manual sobre "Educación Digital Crítica" que recoge un método educativo para poner en práctica los temas desarrollados en esta guía.

Finalmente, partiendo de este nuevo tipo de alfabetización denominada Crítica, podemos definir el concepto de "Lectura Crítica". Según Serrano de Moreno y Madrid de Forero (2007), una persona que lee, desde una perspectiva crítica, debe llegar al sentido profundo del texto y sus ideas, no aceptando las ideas sin discutir, sino con reflexión; prestando atención a las palabras y los enunciados; cuestionando imprecisiones $u$ opiniones contrarias; identificando los diferentes puntos de vista; contrastando con otras alternativas accediendo para ello a otras informaciones; eligiendo las ideas que están de acuerdo con sus ideas y valores; conociendo y manejando el lenguaje para esto; y encontrando el modo de participar en sociedad. Por tanto, para estos autores, la alfabetización crítica consiste en descifrar el código de los textos, participar en la comprensión de los significados, usar textos en diferentes contextos culturales y sociales y analizar críticamente transformando su significado, teniendo en cuenta que los textos siempre representan puntos de vista particulares.

Son varios autoreslos que contribuyen a clarificar este concepto. Raffino (2019) parte del concepto de lectura crítica para ofrecer una serie de ejemplos simples y claros para su entendimiento. Sánchez (2017) se refiere a la importancia de la lectura y su comprensión como punto de partida para el desarrollo del pensamiento crítico, y proporciona herramientas para que el lector lo consiga. Alvarado-Miquilena (2012) establece una diferencia entre lectura crítica y pensamiento crítico. Esta autora define lo primero como la búsqueda de la relectura de textos o mensajes individuales para identificar categorías de discusión, mientras que en el segundo caso, se trata de aprender a pensar de manera autónoma en la creencia de que son las estrategias de interpretación las que apuntan al pensamiento crítico.

Igualmente, podemos citar ejemplos de tipos de lectura crítica en diversos ámbitos o contextos. De este modo, Fainhola $(2003,2006)$ dedica varios de sus artículos a definir la lectura crítica en internet y establecer diferentes propuestas de lectura y evaluación de recursos para ello. Delgado (2018) diseña un programa de lectura crítica en internet para jóvenes con discapacidad intelectual. Díaz Portillo (2008) elabora una guía práctica de lectura crítica de artículos científicos originales en ciencias de la salud a partir de la evaluación de las diferentes partes del texto. Ebevidencia.com (2019) crea un club de lectura crítica colaborativa para profesionales de la enfermería. Salamanca y Sánchez (2013), elaboran un elenco de preguntas para la lectura crítica de los métodos cualitativos de investigación, o Méndez, Arbeláez, Espinar, Gómez y Serra (2014) establecen un estado de la cuestión sobre la lectura crítica en la educación superior.

\section{La responsabilidad de los bibliotecarios sobre la alfabetización crítica}

La Declaración IFLA sobre alfabetización digital, del 18 de agosto de 2017 (IFLA 2017), se refiere a la alfabetización mediática e informativa como un todo, siguiendo el concepto integrador de la UNESCO que ya hemos mencionado con anterioridad. Este documento define la alfabetización digital como la capacidad de aprovechar las herramientas 
digitales, el uso de la tecnología y funcionamiento de internet, con sobrados conocimientos éticos y legales. Por este motivo, se requieren habilidades de alfabetización mediática e informacional y, en consecuencia, crítica, en un proceso de aprendizaje permanente para corregir, sobre todo, la disminución de la confianza en internet, por ejemplo, en el aumento de las noticias falsas o los comportamientos antisociales en la red. IFLA insiste en el compromiso institucional y ético de las bibliotecas para ayudar a los usuarios a acceder a información fiable y auténtica. Considera que debe existir un servicio obligatorio en las bibliotecas para esto y que los gobiernos deben promoverlo como un derecho y asegurar el aprendizaje desde las bibliotecas. Por otra parte, esta Declaración se opone a la censura o bloqueo de contenidos en línea de manera discriminatoria, comprometiéndose con la libertad de expresión.

La alfabetización crítica, como resultado de las alfabetizaciones mediática y digital, se considera esencial para alcanzar los objetivos de la Agenda 2030 de la ONU. La Declaración de IFLA sobre las noticias falsas (IFLA2018), como así aparece en el documento, insta a los gobiernos a no aprovechar la situación para restringir información con la excusa de las noticias falsas y limitar la libertad de expresión de forma injustificada, pero también a invertir en programas de alfabetización informacional y mediática con el componente crítico, apoyar la investigación sobre estas cuestiones y trabajar en colaboración para fomentar el valor de la información digital. Desde el punto de vista de la acción, IFLA propone dar a conocer todos aquellos ejemplos que se dan en bibliotecas de todo el mundo sobre estos aspectos y ella misma está diseñando materiales para ayudar a su comprensión y aportar soluciones, como es el caso de la infografía titulada "Real Solutions to Fake News: How Libraries Help" (IFLA2017). Merece la pena destacar el manual que publica UNESCO (2019) sobre este asunto y el texto sobre el papel de las bibliotecas en un mundo de noticias falsas (Alonso-Arévalo y Martín 2019). Igualmente, el trabajo de Courtney (2017) sobre la colaboración entre periodistas y bibliotecarios en instituciones de educación superior en Irlanda en la era de las fake news.

Además del apoyo de IFLA, son muchos los ejemplos de otras organizaciones que colaboran para la cooperación con las bibliotecas en los diversos tipos de alfabetización. Worldreader (2019) es una organización sin ánimo de lucro (ONL) comprometidos con la lectura global y especialmente con 4 de los ODS de la Agenda 2030: el 4 (educación de calidad), 5 (igualdad de género), 10 (reducir la desigualdad), y 16 (paz, justicia e instituciones fuertes). Desarrolla un programa de lectura en bibliotecas que consiste en proporcionar a las bibliotecas públicas y comunitarias productos de lectura digital según las necesidades potenciales de los lectores, centrándose en contenidos locales y en la formación de los bibliotecarios para la gestión de proyectos de lectura digital. La Fundación Germán Sánchez Ruipérez, tiene una larga tradición en España de investigación en lectura y bibliotecas. En su proyecto "Territorio Ebook" (2019) analiza el impacto del trabajo bibliotecario en la comprensión y motivación de la lectura en internet en lectores digitales de diferentes segmentos de edad, y la experimentación de modelos de club de lectores híbridos.

Otros proyectos de colaboración entre instituciones merecen ser destacados. El proyecto "Plataforma Nubeteca" (2019) surge de la colaboración de La Fundación Germán Sánchez Ruipérez, la Diputación de Badajoz y empresas como ODILO en el marco de Interreg Europa(España-Portugal). Consiste en un proyecto de plataforma para 
lectura colaborativa en el impulso del libro digital y la formación y motivación de lectores "nubetecos". El papel de las bibliotecas públicas es precisamente mantener estos Clubes Nubetecos de lectura digital en bibliotecas. Otros proyectos llevados a cabo en bibliotecas públicas resultan igualmente interesantes. La Biblioteca Pública de Cuenca (2019) en España, organiza los talleres llamados "Familias Conectadas", en los que participan padres, hijos y educadores con el objetivo de favorecer el buen uso de las tecnologías y encontrar soluciones fáciles a problemas concretos. El proyecto "Eliburutegia" (Pulgar 2015), es una apuesta del Gobierno Vasco de España por el mundo del libro digital y las bibliotecas. Consiste en un servicio de lectura que ofrecen las bibliotecas de la Red de Lectura Pública de Euskadi a través de una plataforma de contenidos digitales de uso en varios dispositivos. La participación de las bibliotecas escolares la encontramos en Extremadura a través de su Plan de Educación Digital de Extremadura y el proyecto "Innovated" (2014) para centros educativos sostenidos con fondos públicos, por el que "Librarium", una biblioteca digital está disponible en estos centros para desarrollar programas innovadores de lectura y uso de nuevas tecnologías.

Finalmente, no se cuestiona la importancia de las bibliotecas en la promoción de la
alfabetización en general y en la alfabetización crítica en particular. La responsabilidad
de los bibliotecarios se manifiesta en los documentos de IFLA, en su lucha por el acceso
a una información veraz y fiable, contra la censura y a favor de la libertad de expresión,
componentes éticos fundamentales en ejemplos como el de las "noticias falsas". Esta
responsabilidad se hace patente en la importancia de los proyectos de colaboración de
las bibliotecas con diferentes organizaciones e instituciones que en la actualidad se
están llevado a cabo para contribuir, en general, a la adaptación a la cultura digital y,
en particular, a la alfabetización y aprendizaje a lo largo de la vida.

Finalmente, no se cuestiona la importancia de las bibliotecas en la promoción de la
alfabetización en general y en la alfabetización crítica en particular. La responsabilidad
de los bibliotecarios se manifiesta en los documentos de IFLA, en su lucha por el acceso
a una información veraz y fiable, contra la censura y a favor de la libertad de expresión,
componentes éticos fundamentales en ejemplos como el de las "noticias falsas". Esta
responsabilidad se hace patente en la importancia de los proyectos de colaboración de
las bibliotecas con diferentes organizaciones e instituciones que en la actualidad se
están llevado a cabo para contribuir, en general, a la adaptación a la cultura digital y,
en particular, a la alfabetización y aprendizaje a lo largo de la vida.

Finalmente, no se cuestiona la importancia de las bibliotecas en la promoción de la
alfabetización en general y en la alfabetización crítica en particular. La responsabilidad
de los bibliotecarios se manifiesta en los documentos de IFLA, en su lucha por el acceso
a una información veraz y fiable, contra la censura y a favor de la libertad de expresión,
componentes éticos fundamentales en ejemplos como el de las "noticias falsas". Esta
responsabilidad se hace patente en la importancia de los proyectos de colaboración de
las bibliotecas con diferentes organizaciones e instituciones que en la actualidad se
están llevado a cabo para contribuir, en general, a la adaptación a la cultura digital y,
en particular, a la alfabetización y aprendizaje a lo largo de la vida.

Finalmente, no se cuestiona la importancia de las bibliotecas en la promoción de la
alfabetización en general y en la alfabetización crítica en particular. La responsabilidad
de los bibliotecarios se manifiesta en los documentos de IFLA, en su lucha por el acceso
a una información veraz y fiable, contra la censura y a favor de la libertad de expresión,
componentes éticos fundamentales en ejemplos como el de las "noticias falsas". Esta
responsabilidad se hace patente en la importancia de los proyectos de colaboración de
las bibliotecas con diferentes organizaciones e instituciones que en la actualidad se
están llevado a cabo para contribuir, en general, a la adaptación a la cultura digital y,
en particular, a la alfabetización y aprendizaje a lo largo de la vida.

Finalmente, no se cuestiona la importancia de las bibliotecas en la promoción de la
alfabetización en general y en la alfabetización crítica en particular. La responsabilidad
de los bibliotecarios se manifiesta en los documentos de IFLA, en su lucha por el acceso
a una información veraz y fiable, contra la censura y a favor de la libertad de expresión,
componentes éticos fundamentales en ejemplos como el de las "noticias falsas". Esta
responsabilidad se hace patente en la importancia de los proyectos de colaboración de
las bibliotecas con diferentes organizaciones e instituciones que en la actualidad se
están llevado a cabo para contribuir, en general, a la adaptación a la cultura digital y,
en particular, a la alfabetización y aprendizaje a lo largo de la vida.

Finalmente, no se cuestiona la importancia de las bibliotecas en la promoción de la
alfabetización en general y en la alfabetización crítica en particular. La responsabilidad
de los bibliotecarios se manifiesta en los documentos de IFLA, en su lucha por el acceso
a una información veraz y fiable, contra la censura y a favor de la libertad de expresión,
componentes éticos fundamentales en ejemplos como el de las "noticias falsas". Esta
responsabilidad se hace patente en la importancia de los proyectos de colaboración de
las bibliotecas con diferentes organizaciones e instituciones que en la actualidad se
están llevado a cabo para contribuir, en general, a la adaptación a la cultura digital y,
en particular, a la alfabetización y aprendizaje a lo largo de la vida.

Finalmente, no se cuestiona la importancia de las bibliotecas en la promoción de la
alfabetización en general y en la alfabetización crítica en particular. La responsabilidad
de los bibliotecarios se manifiesta en los documentos de IFLA, en su lucha por el acceso
a una información veraz y fiable, contra la censura y a favor de la libertad de expresión,
componentes éticos fundamentales en ejemplos como el de las "noticias falsas". Esta
responsabilidad se hace patente en la importancia de los proyectos de colaboración de
las bibliotecas con diferentes organizaciones e instituciones que en la actualidad se
están llevado a cabo para contribuir, en general, a la adaptación a la cultura digital y,
en particular, a la alfabetización y aprendizaje a lo largo de la vida.

Finalmente, no se cuestiona la importancia de las bibliotecas en la promoción de la
alfabetización en general y en la alfabetización crítica en particular. La responsabilidad
de los bibliotecarios se manifiesta en los documentos de IFLA, en su lucha por el acceso
a una información veraz y fiable, contra la censura y a favor de la libertad de expresión,
componentes éticos fundamentales en ejemplos como el de las "noticias falsas". Esta
responsabilidad se hace patente en la importancia de los proyectos de colaboración de
las bibliotecas con diferentes organizaciones e instituciones que en la actualidad se
están llevado a cabo para contribuir, en general, a la adaptación a la cultura digital y,
en particular, a la alfabetización y aprendizaje a lo largo de la vida. elemento esencial que debe formar parte, en la actualidad, de los proyectos, planes y políticas de los diferentes gobiernos e instituciones a nivel local, regional, nacional e internacional, ya que se encuentra relacionado con el aprendizaje a lo largo de la vida (lifelong learning) y constituye un componente esencial de todos los tipos de alfabetizaciones que abarca este concepto, en especial, de la alfabetización crítica, tal y como hemos dado a conocer por las diferentes organizaciones implicadas y los diferentes estudios y ejemplos propuestos.

alfabetizaciones, que han ido surgiendo como consecuencia de la evolución de sociedad, pero muestra un interés relevante en las alfabetizaciones mediática y digital, al estar implicados los medios sociales. La lectura crítica, como objeto de la alfabetización crítica, cobra especial importancia en este contexto, debido a las especiales características que toma esta en el entorno digital. 


\section{BIBLIOGRAFÍA}

ALONSO-ARÉVALO, Julio, y Sonia Martín Castilla. 2019. "El papel de las bibliotecas en un mundo de noticias falsas". DesiderataLab. https://gredos.usal.es/bitstream/handle/10366/139437/ Noticias\%20Falsas.\%20DESIDERATA.pdf;jsessionid=F6D0B9BF6A6EC2A660B875162D171ABA? sequence $=1$.

ALVARADO-MIQUILENA, Morella C. 2012. "Lectura crítica de medios: una propuesta metodológica". Comunicar 20 (39): 101-108. https://www.revistacomunicar.com/index.php? contenido=detalles\&numero=39\&articulo=39-2012-12.

Biblioteca Pública de Cuenca. 2019. Familias conectadas. http://reddebibliotecas.jccm.es/intrabibl/ index.php/actualidad/actividades-culturales/item/6522-talleres-familias-conectadas-en-la-bpede-cuenca.

BORGES, Jussara, y Miguel Ángel Marzal García-Quismondo. 2017. "Competencias en información y en comunicación: desarrollo conceptual a partir de la New Media Literacy”. Revista Interamericana De Bibliotecología40 (1) (enero-abril): 35-43. https://www.researchgate.net/ publication/ 318694259_Competencias_en_informacion_y_en_comunicacion_desarrollo_conceptual_a_partir_de_la_New_Media_Literacy.

Carta europea para la alfabetización mediática. 2005. http://www.Euromedialiteracy.eu.

Comisión de las Comunidades Europeas. 2000. Memorándum sobre el aprendizaje permanente. https://uil.unesco.org/i/doc/lifelong-learning/policies/european-communities-amemorandum-on-lifelong-learning.pdf.

COURTNEY, Isabelle. 2017. In a era of fake news information literacy has a role top lay in journalism education in Ireland: an exploratory study of journalism and media faculties and the interactions with their academic libraries. Dublin: MSc, Information and Library Management Dublin Business School. https://esource.dbs.ie/bitstream/handle/10788/3303/msc_courtney_i_2017.pdf? sequence $=1 \&$ is Allowed $=y$.

DELGADO HERRERA, Pedro, Vicenta Ávila Clemente, Inmaculada Fajardo Bravo y Ladislao Salmerón González. 2018. “Un programa de formación en lectura crítica en internet para jóvenes con discapacidad intelectual”. Revista Española de Discapacidad, 6 (II): 229-245. https:// www.cedd.net/redis/index.php/redis/article/view/514/pdf_127.

DESJARDINS, Jérémie. 2000. "Les bibliotèques contre l'Illectronisme”. Bulletin des Biblioteques de France. http://bbf.enssib.fr/consulter/bbf-2000-04-0120-006.

DÍAZ PORTILLO, Jacobo. 2008. Guía práctica de lectura crítica de artículos científicos originales en Ciencias de la Salud. Instituto Nacional de Gestión Sanitaria, 2008. https://www.sepeap.org/wpcontent/uploads/2015/06/Guia_practica_de_lectura.pdf.

EBEVIDENCIA. 2019. Club de lectura crítica colaborativa. Ebevidencia.com. https://ebevidencia.com/ club-de-lectura-critica-colaborativa.

Éduscol: éducation aux médias et à l'internet. 2019. Ministère de l'Education Nationale et de la jeunesse. https://eduscol.education.fr/numerique/dossier/competences/education-aux-medias/ @@document_whole. 
ESPAÑA. 2018. Ley Orgánica 3/2018 de 5 de diciembre de Protección de Datos Personales y Garantía de los Derechos Digitales. BOE, n.294, 6 del 12. https://www.boe.es/buscar/act.php?id=BOEA-2018-16673\&p=20181206\&tn $=2$.

FAINHOLA, Beatriz. 2003. “Lectura crítica en internet”. Lectura y vida. http:// www.lecturayvida.fahce.unlp.edu.ar/numeros/a26n2/26_02_Fainholc.pdf.

FAINHOLA, Beatriz. 2006. "La lectura crítica en internet: evaluación y aplicación de sus recursos". Comunicar 26: 155-162. http://rabida.uhu.es/dspace/bitstream/handle/10272/1274/ b1528024x.pdf?sequence=1.

Fake news and Critical Literacy: the final report of the Commission on Fake News and the Teaching of Critical Literacy in Schools. 2018. London: National Literacy Trust. 2018. https:// literacytrust.org.uk/research-services/research-reports/fake-news-and-critical-literacy-finalreport/.

FASTREZ, Pierre, Philippette Thibault, Julia Bihl y Alice T'kint. 2018. The Media Literacy of Adolescents (13-15 years old): Information search and multimedia creation (2018-2022). https:// uclouvain.be/en/research-institutes/ilc/grems/recherches.html.

FESABID. Grupo de Trabajo de Identidad y Posicionamiento profesional sobre el perfil del consumidor en España. 2014. Tendencias y oportunidades en el consumo de información en la era digital. http://www.fesabid.org/sites/default/files/images/fesabid/llibreFesabid021117.pdf.

GARCÍA-MORENO, María Antonia, y Tony Hernández-Pérez. 2010. "Los retos de la alfabetización informacional en las bibliotecas: guía para supervisar la brecha entre nativos e inmigrantes digitales". Prisma.com 13. https://eprints.ucm.es/12139/1/retos_alfin_biblioteca.pdf.

Grupo Estratégico para el estudio de prospectiva sobre la biblioteca en el nuevo entorno informacional y social. 2013. Prospectiva 2020: las diez áreas que más van a cambiar en nuestras bibliotecas en los próximos años. Madrid: Consejo de Cooperación Bibliotecaria. http:// travesia.mcu.es/portalnb/jspui/handle/10421/7460.

GUTIÉRREZ, Alfonso, y Kathleen Tyner. 2012. Educación para los medios, alfabetización mediática y competencia digital. Comunicar 19 (38): 31-39.http://www.redalyc.org/articulo.oa? id $=15823083005$.

IFLA. 2004. The role of libraries in lifelong learning. Final report of the IFLA project under the Section for Public Libraries. http://www.ifla.org/files/assets/public-libraries/publications/lifelong-learningreport-2004.pdf.

IFLA. 2013. ¿Surcando las olas o atrapados en la marea? Navegando en el entorno en evolución de la información. Percepciones del Trend Report. https://trends.ifla.org/files/trends/assets/ifla-trendreport_spanish.pdf.

IFLA. 2015. Las bibliotecas y la implementación de la Agenda 2030 de la ONU: programa de acción para el desarrollo a través de las bibliotecas. IFLA/ALP. http://www.ifla.org/ES/libraries-development.

IFLA. 2017. Declaración de IFLA sobre Alfabetización Digital. https://www.ifla.org/publications/node/ 11586.

IFLA. 2017. Real solutions to Fake news: How Libraries Help, 2017. https://www.ifla.org/FR/node/ 11584.

IFLA. 2018. Declaración de IFLA sobre noticias falsas. https://www.ifla.org/files/assets/faife/ statements/ifla-statement-on-fake-news-es.pdf. 
Innovated: Plan de Educación Digital de Extremadura. 2014. Extremadura: Consejería de Educación y Empleo. http://bibliotecasescolares.educarex.es/innovated/.

KENDALL, Alex, y Julian Mcdougall. 2012. Alfabetización mediática crítica en la postmodernidad. Comunicar 19 (38): 21-29. http://www.redalyc.org/articulo.oa?id=15823083004.

MÉNDEZ, Juan Camilo, Diana Cristina Arbeláez, Carolina Espinar, Jair Arturo Gómez y Camilo Serra. 2014. "La lectura crítica en la educación superior: un estado de la cuestión". Revista Virtual Universidad Católica del Norte 41 (febrero-abril): 4-18. http://revistavirtual.ucn.edu.co/index.php/ RevistaUCN/article/view/461/983.

Ondula. La tecnología es para las personas. 2019. https://ondula.org/ .

PAÍS VASCO. 2013. Ley 1/2013, de 10 de octubre, de Aprendizaje a lo Largo de la Vida. Boletín Oficial del País Vasco. 199.

PASADAS, Cristóbal. 2010. "Multialfabetización, aprendizaje a lo largo de toda la vida y bibliotecas”. Boletín de la Asociación Andaluza de Bibliotecarios 98-99 (enero-junio): 11-38.

Plan Alba. Junta de Andalucía. 2011. http://www.juntadeandalucia.es/export/drupaljda/ Plan_ALBA09_11_0.pdf.

Plan Estratégico de Aprendizaje a lo Largo de la Vida 2014-2020. 2014. Ministerio de Educación, Cultura y Deporte. Subdirección General del Aprendizaje a lo Largo de la Vida. https:// sede.educacion.gob.es/publiventa/plan-estrategico-de-aprendizaje-a-lo-largo-de-la-vida/ ensenanza/20676.

Plataforma Nubeteca: proyecto biblioteca digital. 2019. Diputación de Badajoz. https://www.dipbadajoz.es/cultura/ceex/index.php?cont=nubeteca.

PULGAR, Francisca. 2015. eLiburutegia: la apuesta del gobierno vasco por el mundo del libro digital. Novagob. https://red.novagob.org/eliburutegia-la-apuesta-del-gobierno-vasco-por-el-mundodel-libro-digital/.

RAFFINO, María Estela. 2019. Concepto de lectura crítica. https://concepto.de/lectura-critica/.

REIA-BAPTISTA, Vítor. 2007. "Hacia una alfabetización en medios: ejemplos en contextos de habla portuguesa". Comunicar 28: 25-31.

SALAMANCA CASTRO, Ana Belén, y Sara Sánchez Castro. 2013. "Lectura crítica de estudios cualitativos (II): preguntas para la lectura crítica”. NURE investigación 66 (septiembre-octubre). http://www.nureinvestigacion.es/OJS/index.php/nure/article/view/649/638.

SÁNCHEZ PRADO, Germán. 2017. "La importancia de la lectura en el desarrollo del pensamiento crítico”. Blog Vicens Vives. https://blog.vicensvives.com/la-importancia-de-la-lectura-en-eldesarrollo-del-pensamiento-critico/.

SÁNCHEZ-GARCÍA, Sandra, y Santiago Yubero. 2015. “Función social de las bibliotecas públicas: nuevo espacio de inserción social”. El Profesional de la Información 24 (2) (mayo-abril): 103-111.

SERRANO DE MORENO, Stella, y Alix Madrid de Forero. 2007. Competencias en lectura crítica. Una propuesta para la reflexión y la práctica. Acción Pedagógica16 (enero-diciembre): 58-68.

STURGES, Paul, y Almuth Gastiner. 2012. "La alfabetización informacional como derecho humano”. Anales de Documentación 15 (1). https://doi.org/10.6018/analesdoc.15.1.147651. http:// revistas.um.es/analesdoc/article/view/147651.

Territorio E-Book: investigación sobre lectura digital. 2019. Fundación Germán Sánchez Ruipérez. https://cds.fundaciongsr.org/portfolio-items/territorio-ebook/. 
UNESCO. Instituto para el Aprendizaje a lo Largo de la Vida. 2015. Directrices para la creación de ciudades del aprendizaje: red mundial de Ciudades del Aprendizaje de la UNESCO, UIL/ME/H/6.

UNESCO. Instituto para el Aprendizaje a lo Largo de la Vida. 2016. Notas sobre política n.6 del UIL: aportes de las bibliotecas para contribuir a las iniciativas nacionales de alfabetización. UIL/PI/H/38.

UNESCO. 2019. Jornalismo, fake news \& desinformação: manual para educação e treinamento em jornalismo. https://unesdoc.unesco.org/ark:/48223/pf0000368647?

fbclid=IwAR1ltj8iF00MPv69hOx4WViYAHzMUlp8VoYlT0Mepi_TYL_utbV5xIgnnEk.

WORLDREADER. 2019. Why Reading. https://www.worldreader.org/why-reading/.

\section{RESÚMENES}

Com base num trabalho teórico de compilação de fontes bibliográficas e descrição dos exemplos mais significativos, apresenta-se uma análise do conceito de literacia crítica no contexto mais amplo da literacia e da aprendizagem ao longo da vida. A literacia crítica é analisada especificamente como um elemento importante da literacia no ambiente digital em que a sociedade actual opera e como uma responsabilidade das bibliotecas. $\mathrm{O}$ conceito de literacia e do seu significado tem vindo a mudar nos últimos anos, verificando-se que a diversidade de termos utilizados para designar uma mesma actividade tem variado ao longo do tempo, em função da evolução das novas tecnologias. Os conceitos de pensamento crítico e leitura crítica são apresentados como componentes fundamentais da literacia crítica num ambiente digital, nos quais todos os tipos de organizações e instituições devem concentrar os seus esforços, conforme os exemplos que aparecem neste texto.

Based on a theoretical work of compilation of bibliographic sources and description of the most significant examples, an analysis of the concept of critical literacy is presented in the broader context of literacy and lifelong learning. Critical literacy is analysed specifically as an important element of literacy in the digital environment in which today's society operates and as a responsibility of libraries. There has been an evolution in the concept of literacy and its meaning in recent years, in which the diversity of terms used to designate the same activity has varied over time, depending on the evolution of new technologies. The concepts of critical thinking and critical reading are presented as fundamental components of critical literacy in a digital environment, in which all types of organizations and institutions must focus their efforts, according to the examples that appear in this text.

\section{ÍNDICE}

Keywords: Digital literacy, information literacy, new media literacy, critical literacy, libraries Palavras-chave: Literacia digital, literacia da informação, literacia em novos media, literacia crítica, bibliotecas

\section{AUTOR}

\section{MARGARITA PÉREZ PULIDO}

Facultad de Ciencias de la Documentación y la Comunicación, Universidad de Extremadura, Badajoz, España. ORCID iD: https://orcid.org/0000-0003-2831-6341. E-mail: marperez@unex.es. 